rera en impôts de l'augmentation considérable de richessc qu'un tel travail ne manquera pas d'entraîner pour le pays.

Conclusions. - En terminant son rapport, la Commission met en évidence tous les avanlages que la Province retirerait d'une tèlle entreprise, et l'urgenec qu'il y a à la réaliser.

La houille noire est rare dans la province de Québec et il est nécessaire, pour le développement de l'industrie, d'augmenter les ressources hydranliques du pays et d'amé liorcr la navigation.

Le projet qui ne comporte qu'une dépense de 6500 ooo $\mathrm{fr}$. permettrail de créer un réservoir d'une caapcité de 4,55 milliards de mètres cubes et d'augmenter la richesse hydraulique utilisable de la rivière d'au moins 600000 HP, donl le prix unitaire ressortirait ì $\mathrm{i}$ fr. 25 le IIP.

On assurerait en même temps au cours d'eau un régimo slable, l'éliminalion des crues désastreuses, au grand avantage de l'jndustric, de la navigation cl du flottage.

H.-L. Bernardin,

Incénieur des Arts et Mamefacturs.

\section{USINE HYDR0-ÉLEGTRIQUE DE CHESTER A HAUTEUR DE CHUTE TRÈS VARIABLE}

L'usine hydro-électrique de Chester est remarquable, non pas par sa puissance, qui est assez modeste, mais bien plutòt par la hauteur de chute réduite sous laquelle elle fonctionne, hauteur de chute d'ailleurs tellement réduite qu'à cerlains moments elle est même complètement nulle : c'est que, en effet, cette usine étant située sur la partie ultime d'une rivière déjà soumise aux influences de la marée, aux variations des niveaux d'amont et d'aval qu'éprouvent habituellement les faibles chutes, viennent s'ajouter à l'aval celles dues au flux et au reflux.

Cette usine, qui vient d'être mise en marche tout récemment, a été construite par la Municipalité de Chester à l'emplacement d'un très vieux moulin qqui utilisait une petite chute de la rivière Dee, chule crée par un petil barragedéversoir construit vers l'an rroo par Ilugh Lupus, comlo de Chester.

La jivière Dee prend sa source dans les montagnes du Nord du Pays de Galles, et vient se jeter dans la mer d'Irlande un peu à l'aval de la ville de Chester, chef-lieu du comté de ce nom. Le lableau ci-dessous indique les valeurss moyennes du débit $Q$ de la Dec, à Chester, et à diverses époques de l'année, ainsi que celles de ha haulemr de chute $I$ ulilisable, abstraction faile des influences perturbatrices de la marée :

Décembre ..................

Janvier, février, mars ...........

Avril, mai octobre, novembre ......

Juin, juillel, aoùl, septembre ......

Minimum minimorum ............

\begin{tabular}{|c|c|}
\hline $\begin{array}{c}0 \\
140 \mathrm{~m}^{3}\end{array}$ & 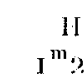 \\
\hline $50 "$ & $1^{\mathrm{m}} \mathrm{g}$ \\
\hline $25 \%$ & \\
\hline $10 \%$ & \\
\hline
\end{tabular}

Sous l'influence de la marée, les hauteurs de chute cidessus indiquées sont notablement réduites, el même, à certaines heures, surtoul en février, mars el avril, au moment dres grandes marées, celles-ci refoulent l'eau d'aval jusque par-dessus le barrage, de telle sorte qu'il n'y a plus de chule du tout, et l'usine cesse de fonclionner. Io service de la distribution de lélectricité rest alors uniquement assuré par lancienme usine ì vapeur.

Le barrage déversoir est établi obliquement en travers de la rivière, el aboutit à l'amout d'une des piles du pont de Chester. L'eau ainsi dévić trarerse le pont el immédiatement à laval de celui-ei, pénète dans la chambre d'eau de la nouvelle usine. En arant de colle chimbre, se trouvent les habituelles grilles el vannes d'irrict.

L'usine comprend trois groupes électrogènes, composis chacun d'une turbine à axe rertical, d'une transmission par engrenages coniques Citroen, à double denture hélicoülale taillée, amplifiant 5 fois la vilesse de rotation, ot d'une dynamo à courant continu à axe horizonlal.

Les turbines sont du type Francis, contripète parallèle a réaction. Elles sont installées directoment dans la chambre d'eau qui forme l'átage inférienr de l'usine. Filles ont áti prérues pour pouvoir fonclionner sous une chule varianl de a a 9 pieds. Deux d'ontre clles onl ćé calculés pour absorber 14,5 mètres cubes par seconde, en lournanl à la vilesse de 50 tours par minule, el devoloppant h15 IIP. L,il

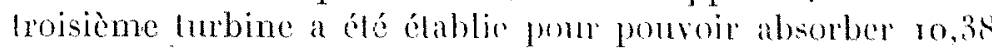
mètres cubes, en tournant à lat vilesse de 5.5 lours ol (nll produisant $305 \mathrm{HP}$. La roue mobile de chaque lurbine est en fonte, avec aubes encastrées cn tòle d'acier. In distribuleur est également en fonte, avec vancltes mobiles commandíes par un régulateur à main. Charque lurbine ces en outre munie d'un lachymètre.

Dans la salle des groupes électrogènes, qui constitur l'élage supérieur de l'usine, se tronve un cadran sur lequel une aiguille indique à chaque instant la hauleur de chule utilisable. A côté des divisions do co cadran indiquanl les hauteurs de chute, se trouvent inserits les nombres de fours correspondants auxquels dojent tourner les lurbines pour obtenir leur rendement maximum.

Les dynamos sont octopolaires, ì excitalion shunt, avec póles auxiliaires pour faciliter la commulation. Elles débitent du courant continu sous 4.10-5oo volts, la régulation du vollage étanl obtenue par varialion du. champ inducteur. Deux de ces dynamos ont une capacité de 235 kilowalls, de tournent en pleine vilesse ì 250 tour's ì la minule; lat troisième dynamo a uno puissance de 185 kilowatls, of lourne i) 2,75 tours.

Signalons en passant l'avantige fue présente le courant continu sur le courant allernatif, pour l'ulilisation des faibles chutes, à hauteurs très variables. On lía pas en effrel à s’inquiéter de la constance de la vilesse en vue du mainlien du synchronisme avec le résenu gínćral, ol l'on peul ainsi faire tourner les turbines at la vilesse correspondant at meilleur rendement.

Voici quelles sont les principales caracléristiques des turbines de l'usine de Chesler' à pleine ouverlure, les hauleurs de chules ulilisables ctant exprinteres an pieds, les debils' an nilliers de pieds cubes par mimule (1), les puissinees on chevaux, a les vilesses normales on lours par minute:

\begin{tabular}{|c|c|c|c|c|c|c|c|c|c|}
\hline HAutburs de CuUte & 1 & is & :B & $i$ & i & f) & $t$ & $x$ & 9 \\
\hline & $10,0)$ & 14,15 & 17,344 & 20,61 & 28,1 & $91,2=$ & 9,20 & 28,0 & 30,00 \\
\hline Puissaned & 15,3 & 43,$2 ;$ & 80,0 & 129,5 & 171,5 & 225,0 & 281,0 & 348,0 & 15,0 \\
\hline Vilesse... & 16,5 & 23,0 & 28,5 & 23,0 & 37,0 & 40,5 & 43,5 & 40,0 & 50,0 \\
\hline$\theta$ & 7,38 & 10 , 保 & 12,80 & $|A, \vec{I}|$ & $16, n, 7$ & $18,(12)$ & 10,50 & 20,85 & 92,00 \\
\hline (5) Puissance & 11,1, & 32,0 & 68,8 & 90,0 & 127,0 & 105,5 & 209,0 & $24,2,0$ & 305,0 \\
\hline Vitoss & 18,6 & 250 & 31,6 & $36 \pm$ & $\$ 1,0$ & 45,0 & $18, \pi$ & 51,0 & $\pi$ \\
\hline
\end{tabular}

Le conant produil par les frois dymames passe d'abord

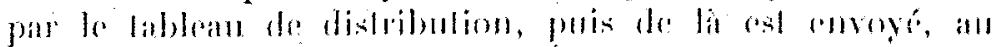
moyen de trois cables armes, it ine sons-station pour das-

(1) Je pied anglais vaut 0 mo4s et to pied cube 28,916 litres. 
servir le quartier roisin de celle-ci. Celte sous-station est d'ailleurs reliée à la station à vapeur de New Crane Street, qui alimentait Ja ville jusqu'ici, et où sont installés un survolteur-dévolteur ainsi qu'une batteric-tampon de 3 ooo ampère-houres.

C'est M. S.-E. Britton, l'ingénieur. élcotricien de la ville de Chester, qui eut l'idéc d'uliliser la chule du moulin de la Dee. Celte idée, émise en plein pays de houille noire, ne ful pas sans susciter de vives critiques, mais son auteur, sans se décourager des oppositions qu'il rencontrait, dressa un projet, et le présenta à la "Corporation " de Chester qui le soumit à l'avis de deux ingénieurs-conscils, MM. Iurlzig et J.-S. Wilson. Ceux-ci estimèrent la dépense de promier établissement à I $_{2} 500 \mathscr{f}$, et les dépenses annuelles ì I $3.25 \mathfrak{f}$. Ils évaluèrent en outre la puissance produitc dans le courant d'une année à un peu plus de un million de kilowatt-heures, ce qui, au taux de $x$ penny lc kilowatiheure, devail produire $4255 £$. Défalcation faile des charges, il devait donc rester un bénéfice annuel de $2930 £(75000$ francs en nombre rond), soit 23 , 亿 \% du capital engagé. Ia proposition de $M$. Brilton paraissail donc intéressante, aussi fut-ellc adoptée.

II. BELLET,

\section{REVUE IES SOCIÉTÉS SAVANTES ET DES PUBLICATIONS TEGHNIQUES}

\section{ACADÉMIE DES SCIENCES}

\section{SISMOLOGIE}

Tremblements de terre destructeurs et précipitations atmosphériques. Note de M. DE Montessus de Balcore, séance du 14 avril 1943.

Los innonbrables statisliques exéculées dans le bul de savoir si lit répartilion annuelle des tremblements de terre présente on non quelque relation avec les mois ou les saisons, se rapportent presque cxclusivement aux mois ou aux suisons astronomiques, quoique leur's auleurs aient cherché plus ou moins explicitement à démonLrer ou à nicr des relations d'ordre climatérique. Dans l'un el l'autre cas, le but réellement visé a donc été manqué. Or, les précipilations atmosphériques sont le phénomène clinatérique principal auquel on puisse plausiblement attribuer un rôle sismogénique, en conséquence de la théorie très répandue des tremHements de terre d'eflondrement dus, prétend-on, à la circulation des caux souterraincs qui dissolvent et enlèvent les couches terrestres plus ou moins profondes. Pour résoudre définitivemeni la question, d'ailleurs tròs controversée, il faul l'aborder directement, ec qui n’a jamais été l'ait.

Dans ce but, l'auleur a pris le Catalogue des 4 I36 tremblements de terre destructcurs de J. Milne et mis en parallèle chacun d'entre eux avec les précipitations des pays où ils ont eu leur contre, quand il est assez bien déterminé, en se basant sur ] I Mappemonde climatérique publiée par de Martonne dans sa Góographie physique.

Sant quelques anomalies sans importance fondamentale, les courbes annuclles de précipilations établies par mois se ramènent à un are de sinusoüde, l'intervalle des maximums et des mininums mensucls oscillant au plus entre 3 et 5 mois. Au contraire, Jorsque les courbes des nombres mensuels de séismes par climats se ramènent a cette forme, ce qui est assez rarement possible, les maximums et les minimums sont séparés par des interralles quelconques cl peuvent même se présenter en 2 mois consécutifs. La confrontation mensuelle serail donc illusoire, el il convient de l'útablir par trimestre. En voici les résultats obtenus en décalant raturellement de 6 mois tout ce qui sc rapporte ì l'hémisphère austral :

t. Lo maximum sismique tombe dans le mène trimestre que la maximum de précipitations. Climats : mandehourien, océanien, péruvien, soudanien, 527 séismes.

a. T.e minimum sismique tombe lans le meme trimestre que le minimum de précipitations. Climats : amazonien, breton, mexicain, ukrainien. 836 séismes.

An lolal, rafiz séismes semblent se conformer à une relation directe cntre les demx ordres de phénomenes, incomplètement loutefois, puisque da coïncidence n'a lien que pour un seul des deix éléments de comparaison, maximum on minimum.

3. Le maximun sismique tombe dans le même trimestre que le minimun de précipitations, Climats : andin, parisien, pendjabien, a25 séismes.
4. Le minimum sismique lombe dans te meme trimestre que le maximum de précipitations : Cilimals : arctique, damubien, indon, norvégien, portugais, polonais, polynésien, saharien, sénégalien, siamois, syriaque. 1783 séismes.

Au tolal, 2008 séismes semblent. se conformer incomplìtement aussi, à une relation telle que l'effet sismogénique des précipitations ne se produirait qu'environ 6 mois plus tard, ce qui serail contradictoirc arec le résultat correspondant anx denx premiers cas.

5. Ni le maximum, ni le minimum sismique ne tombent dans le même trimestre que le maximum ou le minimum de précipitations, ni inversement. Climals : arabien, bengalien, chinois; hellène, sibérien. 653 séismes.

6. Dans aucun cas, le maximum et le minimum sismique ne tombent à la fois dans le môme trimestre que le maximum et le mininum de précipitations, ce qui correspondrait à la dépendance mutuclle.

7. Dans aucun cas, le maximum el le minimum sismique ne tombent à la fois dans le mème trimestre que le rninimum ct le maximum de précipitalions, ce qui correspondrait à une dépendance mutuelle retardée de 6 mois.

8. Les différents climats, qu'ils soient riches ou pauvres en séismes ou en précipitations, se rópartissent au hasard dans les cinq premières catégories.

9. En général, pour un climal déterminé, si l'on exprime en pour roo du total correspondant de séjsmes les nombres de séismes du trimestre le plus riche el le plus pauvre, leur différence est L'autant plus petite, que le nombre total correspondant de séismes est plus grand; et il en est de môme pour les nombres mensuels. D'après la loi des grands nombres, c'est bien là le critérium de l'absence de toule dépendance entre les deux ordres de phónomènes.

En résumé, il n'y a aucune relation de cause à effet entre les tremblements de terre destructcurs ct les précipitations atmos. phériques, et cela ne présente aucun intérêt de constater par exomple que tel grand événement sismique a suivi une période d'inondations, ainsi qu'on l'a souvent fait.

\section{ÉTUDES GÉOLOGIQUES}

L'histoire fluviale et glaciaire de la vallée du Rhone aux environs de Lyo?. Notes de M. Charles Depéretr. (Séances des 6 et 13 octobre 1913).

Peu de vallées françaises ont suscilé d'aussi nombreux travaux que la vallée du Rhône aux environs de Lyon. Pour se limiter aux grandes étapes de l'étude de ces formations fluviales et glaciaires, on doil citer la belle monographice de Falsan ef Chantre ( 1879 ) où sont précisées les limites du glarier rhodanien et la direction de ses courants d'écoulement. En 1884 , Fontamnes établit la distinction capitale des alluvions ferrugineuses des plateaux, duge pliocène et des allurions grises quaternaires, celles-ci emboîtées 